\title{
Progress in the Numerical Solution of the Nonsymmetric Eigenvalue Problem
}

\author{
Zhaojun Bai \\ Department of Mathematics, University of Kentucky, Lexington, KY 40506, USA
}

With the growing demands from disciplinary and interdisciplinary fields of science and engineering for the numerical solution of the nonsymmetric eigenvalue problem, competitive new techniques have been developed for solving the problem. In this paper we examine the state of the art of the algorithmic techniques and the software scene for the problem. Some current developments are also outlined.

KEY WORDS nonsymmetric matrices; sparse matrices; eigenvalue problem; EISPACK; LAPACK

\section{Introduction}

Having worked several years on the LAPACK project [2] and having communicated with a variety of users who work in diverse fields involving scientific computing, the author has seen a growing demand for the numerical solution of the eigenvalue problems. Since the publication of Parlett's exploratory review paper, 'The software scene in the extraction of eigenvalues from sparse matrices' [35], nearly a decade ago, many new numerical methods and analyses have been developed for the eigenproblem. The aim of this essay is to review the origins of the eigenvalue problem and the progress of the numerical techniques for the problem over the past decade and to share our view and expertise within the scientific computing community.

The survey is by no means complete. One reason for this is that relevant articles may be found scattered throughout the scientific and engineering literature, and the task of tracking them all down is impossibly large. The author apologizes for his ignorance of any important contributions to the problem that are not mentioned here. A new book by Saad [44] is an elegant source for studying the state of the art in large eigenproblem techniques. This review will only focus on the nonsymmetric eigenvalue problem in the aspects of its origins, algorithmic techniques, software scene and work in progress. 
As defined by Parlett [35] a decade ago, there are two different user groups for the eigenproblem. One is called the intensive user group and the other called the sporadic user group. Spectral analysis is imperative to the work of the former group; they have been expending tremendous efforts in term of time and funding in order to extract the desired spectral information. But, for the latter group, the need to compute eigenvalues arises occasionally and the user wants to obtain them with minimal fuss.

With the rapid advances of computer facilities, in particular, the massively parallel computers, and the new engagement of interdisciplinary scientific computing activities, as proposed by J. W. Demmel, there are two different camps in each user group according to their different desired priorities in terms of computation details, reliability and execution time of a program. The first camp is made up of traditional library users and the second camp is made up of high performance computing researchers. For the first camp the desiderata can be characterized as follows:

(1) easy user interface with hidden computation details,

(2) reliability: the code should fail as rarely as possible,

(3) execution time.

However, for the second camp, the desiderata are

(1) execution time,

(2) being able to access to internal details to fine-tune data structures to their applications,

(3) reliability: a program should expend only a negligible amount of time, space or code in checking or taking precautions against rare eventualities that the user knows may never arise for his or her particular applications.

These different desiderata give an extra dimension to numerical algorithm development and analysis. To what extent can we satisfy both camps? In this essay we will try to address this interesting question with respect to the nonsymmetric eigenvalue problem.

There are no clear boundaries for a problem being small and large and a matrix being dense and sparse. All of them are relative and are changing rapidly with the advances of algorithms and computer technology. A matrix of the order of a couple of hundred used to be considered large, but on today's high performance workstations and supercomputers it is small. The list of today's performance and benchmarks for an algorithm on highperformance computers often starts with matrices of the order of above a couple of hundred and up to thousands. A matrix is considered to be sparse if only a small percentage of its entries are nonzero. However, concerning the tradeoff between the sparsity and algorithm complexity and performance, we do not necessarily exploit the zeros. Following Parlett's suggestion in his 1984 paper [35], 'it is vital to science that one not be more precise than is necessary for the purpose in hand', we will leave the user to decide whether his or her problem is small or large, dense or sparse.

Finally, to numerical analysts it is well known that the techniques for treating dense and sparse matrices, small and large problems are closely related. An inner loop of a method for large and sparse eigenproblem often constitutes a small and dense problem. The small problem solver could be a bottleneck for the large problem.

The rest of the paper is organized as follows. Section 2 reviews the main features of the nonsymmetric eigenvalue problem solvers in the LAPACK package. Sections 3 and 4 discuss the cases where the users want to have more than what the LAPACK package can offer. Section 5 describes algorithms and software for the sparse nonsymmetric eigenvalue 
problem. Section 6 discusses some contributions in the last decade which have become more widely appreciated. Sections 7 and 8 describe some work in progress and conclusions.

\section{The nonsymmetric eigenproblem in LAPACK}

LAPACK [2] is a transportable public domain library of Fortran 77 subroutines for solving the most common matrix computation problems, such as solving linear systems, least squares problems, eigenproblems and so on. The library was mainly supported by NSF beginning in 1987. It is a further development of the successful LINPACK and EISPACK libraries $[15,48,22]$ that were developed in the 1970s. A great deal of effort has been expended to improve accuracy and robustness, to add new functionality, and to incorporate design methodologies and algorithms that make LAPACK appropriate for today's high performance shared memory vector and parallel computer architectures. The design, implementation and performance evaluation of LAPACK has attracted significant interest from user communities and from computer manufacturers.

LAPACK is said to be 'transportable' rather than 'portable', because the fastest performance requires that highly optimized block matrix operation already be implemented on each machine by the manufacturer or someone else. Nevertheless, the algorithms used in LAPACK are so well specified and understood that they have been turned into 'black box' programs demanding no foreknowledge of where the eigenvalues are likely to lie and no tricky choices of tolerances or accuracy.

There are three levels of subroutines in LAPACK ${ }^{1}$ for addressing the standard nonsymmetric eigenvalue problem:

1. Driver routines for the nonexpert and for the expert: These drivers solve a complete problem. The user can choose to compute the eigenvalues and left and/or right eigenvectors, or the Schur decomposition. In addition, with the expert drivers a balancing transformation can be used to improve the conditioning of the eigenvalues and eigenvectors; the user can choose to compute condition numbers for the eigenvalues and the right eigenvectors, to order the eigenvalues on the diagonal of the Schur form, and to compute a condition number for the average of the selected eigenvalues and for the right invariant subspace corresponding to the selected eigenvalues.

2. Computational routines: These subroutines perform distinct computational tasks, such as Hessenberg reduction, the Schur decomposition of a Hessenberg matrix, inverse iteration for selected eigenvectors, and condition number estimation.

3. Auxiliary routines: Auxiliary routines in this level perform relatively low-level operations, such as unblocked Hessenberg reduction, swapping the adjacent diagonal (block) entries in Schur form, solving 2 by 2 eigenproblems and so on.

These LAPACK subroutines are not just more efficient updates of their popular predecessor EISPACK. In order to obtain the better performance of LAPACK subroutines on a wide range of shared-memory vector and parallel processors, some new algorithms have been designed to fit the memory access patterns with respect to the multilayered memory hierarchies of the machines. For example, a block Hessenberg reduction algorithm has been adapted in LAPACK for reducing the original matrix a condensed form by orthogonal transformation.

${ }^{1}$ Individual routines from LAPACK can be obtained by electronic mail through netlib: netlib@ornl.gov or netlib@research.att.com. The complete package can be obtained on magnetic media from NAG at Jordan Hill Road, Oxford OX2 8DR, England. 
Following the reduction to condensed form, a multishift $\mathrm{QR}$ algorithm has been developed in order to using block matrix operations. The traditional EISPACK routine HQR uses a double shift; the multishift strategy uses block shifts of higher order and can perform matrix-vector operations instead of vector-vector operations and also obtains modest speedup.

Along with the new goals of LAPACK in terms of stability, efficiency, accuracy and robustness, LAPACK also provides much wider functionality for the nonsymmetric eigenvalue problem, including computing the Schur form and Schur vectors, ordering selected eigenvalues in Schur form, and computing right and/or left eigenvectors. Last but not least, with the new condition number estimation techniques we can produce condition numbers along with the basic solutions. This is a valuable byproduct of the successful perturbation theory conducted by many numerical analysts over three decades.

All LAPACK subroutines have been through massive testing and performance evaluation on a large class of modern computers with help of over 40 test sites from USA and Europe. The critiques and comments from the potential user communities and computer vendors have improved the programs in many aspects.

With the large and fast-growing storage capacity of today's high-performance computers, the domain of the LAPACK grows. The author has used a LAPACK driver routine to find all the eigenvalues of a 2000 by 2000 matrix - a computation that would have been difficult or impossible 10 years ago. It took about 575 seconds of CPU time on a CRAY-2 machine.

Does LAPACK satisfy all users' needs? Who will remain unsatisfied with LAPACK for their applications? What has been missed in LAPACK? We will try to address these questions in the following sections.

\section{Why we remain unsatisfied with LAPACK}

Without a doubt LAPACK subroutines will be used extensively in the coming years. For those sporadic and traditional library users of eigenvalue solvers whose matrices can be stored in-core and who think reliability is more important than execution time, LAPACK subroutines are their desired choice. However, for those intensive users and high-performance competitors, LAPACK does not provide all they are looking for. The following are the main complaints on the nonsymmetric eigenproblem routines.

The penalty of exception handling: The speed of some subroutines is penalized by spending a considerable amount of time, space, or code checking for rare events. If the eigenvalue computation is the inner loop of some large computation, such a penalty could significantly slow the computation, even though the users (who are more interested in speed) know that their problems are very well-conditioned. For example, in order to resist the possible overflow, we cannot use the standard Level 2 BLAS triangular solver in computing the eigenvectors of a triangular matrix and in inverse iteration for the selected eigenvectors, because there is no protection against overflow in the BLAS routine. Instead, we had to develop another much more complicated triangular solver including sophisticated scaling in the inner loop: it has 300 lines of Fortran versus the standard Level 2 BLAS code with 159 lines of Fortran (not including comments). This gives us a double performance penalty, since we cannot use optimized BLAS, and since we must do many more floating-point operations and logical tests.

Without partial eigenvalue handling: There are eigenvalue problem applications where the users are only interested in the eigenvalues and their corresponding invariant subspace in a specified region of the complex plane, such as in a vertical strip including the imaginary 
axis. However, the LAPACK routines always compute all eigenvalues of the matrix. The users have to sort them out by themselves.

Single data structure and no sparsity handling: The main drawback to LAPACK is that it does not try to exploit sparsity in the original matrix. A square matrix is stored in essentially one standard, conventional, two-dimensional data structure. Many matrices arising in practice are too large to be stored in-core. In this case it is necessary to take advantage of the sparsity of the original matrix to reduce memory requirements and computation time. In the next section we will examine the origins of large-scale eigenvalue problems in science and engineering to illustrate the need for exploiting the sparsity of the matrix.

\section{Where are the origins of large eigenvalue problems}

For a small matrix eigenproblem the algorithm and software are in such good standing that we can solve the problem efficiently without the need to know where it comes from. However, for a large problem, algorithm development still is a research subject. Therefore, before we consider an algorithm for such a problem, it does help to know where it came from and what users really want.

In [35] Parlett discusses eigenproblems from structural engineering, quantum chemistry, and plasma physics. Most of them are symmetric. In addition, Markov chain modeling and numerical simulation of certain chemical reactions (see Stewart [52] and Saad [41]) have become the standard test problems for numerical methods. In this section we will examine some newly arising nonsymmetric eigenvalue problems coming from the scientific computing community.

\subsection{Power system stability study [34]}

Low-damped electromechanical oscillations are a common phenomenon in modern electric power systems. The damping of these oscillations is dependent on system structure, operating conditions, and the effects of automatic-controller action. An efficient way to combat these oscillations is through the installation of additional signals to the generator excitation systems. In recent years this area of work has led to the development of numerical methods to determine the source of these oscillations and obtain solutions via excitation control.

The power system stability problem is represented by a set of differential algebraic equations. If we write the Jacobian matrix of the entire set of equations evaluated at an operating point as

$$
J=\left(\begin{array}{ll}
J_{1} & J_{2} \\
J_{3} & J_{4}
\end{array}\right)
$$

then the power system state matrix is defined as

$$
A=J_{1}-J_{2} J_{4}^{-1} J_{3}
$$

The dominant eigenvalues of the matrix $A$ determine the stability of the nonlinear system. In practice, the matrix $J$ is very large and sparse, but the state matrix $A$ is not sparse. The problem is to calculate the desired eigenvalues of $A$ without forming it explicitly. A group of researchers under the leadership of Martins [34] is developing an efficient algorithm on an Intel distributed memory machine. 


\subsection{Numerical analysis of optical waveguides [47]}

In recent years an accurate numerical analysis for optical waveguides has become of practical interest for optimum design of optical devices and optical integrated circuits. Under certain conditions the propagation constant $\beta$ and electric field $\phi(x, y)$ in the waveguide are described by the scalar Helmholtz equation of the form

$$
\left(\nabla^{2}+k^{2} n^{2}(x, y)-\beta^{2}\right) \phi(x, y)=0
$$

where $k$ is the wave number and $n(x, y)$ is the refractive index. The finite difference discretization on a nonuniform mesh converts the equation into an eigenvalue problem

$$
A \phi=\beta^{2} \phi
$$

where $A$ is a banded matrix, and $\phi$ is a column vector whose components represent the electric field in each cell. The eigenvalues corresponding to the bound modes range between the refractive index of the channel layer and the refractive index of the cladding layer. In other words, only those eigenvalues with their real parts in a certain interval are of interest.

A recent report by Galick et al. [21] presents a numerical approach to the simulation of a dielectric channel waveguide and is based on the solution of an eigenproblem for the two transverse components of the magnetic field.

\subsection{Navier-Stokes solver}

The eigenvalue problem is often associated with the stability analysis of a complex flow governed by the Navier-Stokes equations.

The stability analysis of viscous free surface flow [11]: Slide coating is an industrial precision operation. A liquid film is formed on an inclined plane, flows down and off the plane and onto a moving sheet, displacing air as it wets the smooth solid surface of the sheet. The desired liquid flow is steady stable and uniform across the width of the sheet and is governed by the Navier-Stokes equations for two-dimensional, incompressible flow. Under the momentum conservation, mass conservation and kinematic boundary conditions and by means of Galerkin's method and finite element basis functions, one has a set of nonlinear differential algebraic equations of the form

$$
f(y, \dot{y}, p)=0
$$

where $y$ is the vector of all nodal unknowns, i.e., the coefficients of the finite basis element basis functions, and $p$ is the vector of physical and discretization parameters. The prototype problem examined in [11] is the asymptotic stability of the steady state to infinitesimal disturbances. Small disturbances $y_{1}$ imposed on a steady state $y_{0}$ are governed by the linearized equation (up to the first order, see [11])

$$
f_{\dot{y}}\left(y_{0}, 0, p\right) \dot{y}_{1}+f_{y}\left(y_{0}, 0, p\right) y_{1}=0
$$

Then $y_{1}$ is given by

$$
y_{1}(t)=\sum_{i=1}^{N} \epsilon_{i} \exp \left(\lambda_{i} t\right) x_{i}
$$


where $x_{i}$ is a generalized right eigenvector associated with eigenvalue $\lambda_{i}$ of the eigenvalue problem

$$
J x=\lambda M x
$$

where $J=f_{y}\left(y_{0}, 0, p\right)$ and $M=-f_{\dot{y}}\left(y_{0}, 0, p\right)$, and $\epsilon_{i}$ is the component of $y_{1}$ along $x_{i}$ at $t=0$. Typically, $J$ and $M$ are of order 2000 to 4000 , nonsymmetric, banded, sparse and singular. A steady state is stable if all eigenvalues have negative real parts. The computational task is to find the 'dangerous eigenmode', i.e., the eigenvalue with the largest real part.

Instability of ferrofluids: Recently, Boudouvis et al. [8,9] have reported the stability analysis of three-dimensional patterned states in the normal field instability of ferrofluids. The size of the related eigenvalue problem, when all possible patterns are 'allowed' (by the boundary conditions) to compete, is of the order of 100000 !

Perturbation analysis of unsteady, transonic, viscous flow over airfoils: The eigensystem analysis is associated with small perturbation of a finite different representation of the Navier-Stokes equations for analyzing complex flows such as unsteady, transonic, viscous flow over airfoils. Such eigensystem information is used to reduce the number of time steps required to reach a steady solution and accelerate the convergence [19] and to determine the modal behavior of fluid in a fluid-structure interaction problem [33]. The matrices involved in such problems are very large (up to $24000 \times 24000$ ), real, banded sparse and unsymmetric. The interesting eigenvalues are the ones with small negative real parts, or the dominant ones.

\subsection{Others}

In [23] an eigenproblem solver is required for the numerical detection of a Hopf bifurcation of a parameter-dependent nonlinear system modeling a tubular reactor. Chatelin et al. [10] show an eigenproblem from aeronautical industries. The interesting eigenvalues are those whose imaginary part lies in a frequence range chosen by engineers. The matrix is sparse with a block structure and has order $10^{3}$ to $10^{4}$. In [36] an interesting eigenproblem of a transfer matrix from the Ising model used in chemistry and biology is discussed. The matrix is nonsymmetric, very sparse (only having two entries at each column and row) and huge (of the order of $2^{n}$ ). The desired eigenvalues are the two largest ones.

\section{Software for the sparse nonsymmetric eigenproblem}

There has been significant progress for the large symmetric eigenvalue problem over the last two decades. The Lanczos algorithm has become the most favored algorithm. Today you can get the software through public domain or commercial packages. However, the large nonsymmetric eigenvalue problem is still a research topic. We are still trying to understand better the problem, the existing algorithms and their numerical behaviors.

In this section we review progress in the numerical solution of the problem in the past decade. We focus on three popular algorithms which are mostly studied in the numerical analysis community. We also list information on software which has been developed mostly by numerical analysts, but it should be noted that a massive testing phase has not been applied to the software. Some of the software still consists of research codes.

In sparse matrix computations it is widely accepted that it is important to reference the matrix in question only through a user-provided subroutine for forming matrix-vector 
products. All algorithms and software discussed in this section have such a feature. In some applications this essential operation could be the major cost of the overall computation. The exploitation of the structure and sparsity of the matrix is passed to the users. The performance of these operations on supercomputers can differ significantly from one problem data structure to the others.

The author should note that besides the three popular methods discussed in this section, there are some other methods which have not been reviewed, such as the GoldhirschOrszag-Maulik method [25] proposed for solving the hydrodynamic and mechanical stability analysis and the generalization of the popular Davidson's method for a general matrix used in computational chemistry [13]. Sad to say, not much attention has been paid to these algorithms in the numerical analysis community.

\subsection{Algorithms}

This section gives a brief description of the main ideas of the three popular methods. There is an extensive literature on these methods, too much to be listed in this essay. The reader is referred to $[26,44]$.

The simultaneous iteration method, whose prototype is Bauer's Treppeniteration, is an extension of the power method. It is still one of the most popular methods used for computing the dominant eigenvalues of large matrices, in particular, in structural engineering. The iteration starts with an $n \times m$ matrix $Q_{0}$ and generates a sequence of $n \times m$ matrices $Q_{k}$ according to the formula

$$
A Q_{k-1}=Q_{k} R_{k}
$$

where $R_{k}$ is a nonsingular matrix chosen variously by different implementations. Since the column space of $Q_{k}$ and $Q_{k} R_{k}$ are the same, $R_{k}$ can be regarded as a scaling factor. Then one forms a projected matrix: $B_{k+1}=Q_{k}^{T} A Q_{k}$, and reduces it to $B_{k+1}=Y_{k} T_{k} Y_{k}^{-1}$, which is an eigendecomposition or Schur decomposition of $B_{k+1}$; finally $Q_{k}$ is overwritten by $Q_{k} Y_{k}$. It has been proved that the rate of convergence of the $i$ th column of $Q_{k}$ depends on the ratio $\left|\lambda_{m+1} / \lambda_{i}\right|$, where we assume that the eigenvalues $\left\{\lambda_{i}\right\}$ of $A$ are ordered so that $\left|\lambda_{1}\right| \geq\left|\lambda_{2}\right| \geq \ldots \geq\left|\lambda_{n}\right|$.

The following Arnoldi and Lanczos methods are both in the class of Krylov subspace techniques. Members of this class generate (bi-)orthogonal bases for Krylov subspaces $K_{m}(q, A)=\left\{q, A q, A^{2} q, \ldots, A^{m-1} q\right\}$ (and $K_{m}\left(p, A^{T}\right)$ ) with the given initial vector(s) $q$ (and $p$ ), and then compute eigenvalues of a reduced matrix obtained from $A$ by restricting $A$ to the Krylov subspace. A subset of the computed eigenvalues is selected as approximations to the eigenvalues of the original matrix. Specifically, the Arnoldi algorithm first produces an orthonormal basis of the Krylov subspace $K_{m}(q, A)$, which can also be regarded as a truncated reduction of an $n \times n$ matrix $A$ to upper Hessenberg form. Starting with a given vector $q_{1}$ of $\left\|q_{1}\right\|_{2}=1$, after $m$ steps we have

$$
A Q_{m}=Q_{m} H_{m}+h_{m+1, m} q_{m+1} e_{m}^{T}
$$

where $Q_{m}^{T} Q_{m}=I, Q_{m}^{T} q_{m+1}=0, H_{m} \in \mathbb{R}^{m \times m}$ is an upper Hessenberg matrix. Then the eigenpairs $\left\{\left(\theta_{i}, z_{i}\right)\right\}$ of the reduced matrix $H_{m}$ are computed. The approximate eigenpairs of $A$ are selected from the so called Ritz pairs $\left\{\left(\theta_{i}, Q_{m} z_{i}\right)\right\}$.

The Lanczos process is a procedure to generate the biorthogonal bases of the Krylov subspaces $K_{m}(q, A)$ and $K_{m}\left(p, A^{T}\right)$ that can be regarded as a truncated procedure for the tridiagonalization of a general matrix. In matrix notation, starting with given vectors $p_{1}$ and 
Table 1. Spectrum transformation

\begin{tabular}{lll}
\hline Transformation functions & Operation & References \\
\hline Polynomial $p(x)$ & $p(A) v$ & {$[41]$} \\
Shifting-and-inverting $(x-\mu)^{-1}$ & $(A-\mu I)^{-1} v$ & {$[37]$} \\
Cayley transformation $c(x)=(x-\alpha) /(x+\alpha)$ & $(A+\alpha I)^{-1}(A-\alpha I) v$ & {$[7,11,23]$} \\
Rational function $r(x)=p(x) / q(x)$ & $r(A) v$ & {$[38,39]$} \\
Exponential function $e^{x}$ & $e^{A} v \approx p(A) v \approx r(A) v$ & {$[19,11]$} \\
\hline
\end{tabular}

$q_{1}$ and $q_{1}^{T} p_{1} \neq 0$, after $m$ steps we have

$$
\begin{aligned}
A Q_{m} & =Q_{m} T_{m}+\beta_{m+1} q_{m+1} e_{m}^{T} \\
A^{T} P_{m} & =P_{m} T_{m}^{T}+\gamma_{m+1} p_{m+1} e_{m}^{T}
\end{aligned}
$$

where $P_{m}^{T} Q_{m}=D_{m}, D_{m}$ is a diagonal matrix. There are certain free choices in the procedure to force $D_{m}$ to be identity matrix, or $\left\|p_{i}\right\|_{2}=\left\|q_{i}\right\|_{2}=1$. Then eigentriplets $\left\{\left(\theta_{i}, z_{i}, w_{i}\right)\right\}$ of the reduced matrix pencil $\left(T_{m}, D_{m}\right)$ are computed, The approximate eigentriplets of $A$ are selected from the Ritz triplets $\left\{\left(\theta_{i}, Q_{m} z_{i}, P_{m} w_{i}\right)\right\}$.

We should note that for the simultaneous iteration or the Arnoldi method, a subroutine to generate the vector $y=A x$ for a given vector $x$ has to be provided. However, for the Lanczos method subroutines to generate both the vectors $y=A x$ and $z=A^{T} x$ have to be provided.

Both Arnoldi and Lanczos algorithms can be 'blocked', i.e., work with several vectors instead of a single vector during the projection process, just as for the simultaneous iteration. Blocking is a particularly appealing feature in a large out-of-core problem for exploiting the presence of a block of the matrix $A$ in fast memory as much as possible. Some preliminary work of block Krylov subspace methods has been presented in [44,46,45].

\subsection{Spectrum transformation}

Unfortunately, each algorithm favors a particular part of the spectrum which may not be what the user wants. Simultaneous iteration favors the dominant eigenvalues, whereas the Arnoldi and Lanczos algorithms prefer the outer part of the spectrum. This is where the spectrum transformation comes into the picture. The spectrum transformation strategy (sometimes called preconditioning techniques) is to transform the desired eigenvalues in the complex plane toward the favored part of the chosen algorithm in the hope of accelerating the convergence rate and separating the desired eigenvalues from the undesired ones, leading to better conditioning.

Many different spectrum transformation techniques have been tried over the years. Table 1 lists some of the known spectrum transformation functions, the matrix-vector operations involved and references. It is clearly a problem-dependent strategy; the user's knowledge of where eigenvalues are likely to lie can be used to choose the transformation function.

\subsection{Software}

Table 2 summarizes available software for the large nonsymmetric eigenvalue problem, and the author apologizes for his ignorance of any other existing good implementation of the 
Table 2. Software for sparse nonsymmetric eigenvalue problems

\begin{tabular}{llllll}
\hline Authors & Year & Name & Method & Lines & References \\
\hline Stewart-Jennings & 81 & LOPSI & Simul. iter. & 1054 & {$[53]$} \\
Stewart-Bai & 78,91 & SRRIT & Simul. iter. & 2417 & {$[52,6]$} \\
Duff-Scott & 91 & EB12 & Simul. iter. & 3091 & {$[18]$} \\
Saad & 85 & ARNLS & Arnoldi & 1783 & {$[42]$} \\
Saad & 86 & ARNINV & Arnoldi & 2619 & {$[42]$} \\
Sorensen & 91 & ARNUPD & Arnoldi & 2743 & {$[49]$} \\
Cullum-Willoughby & 86 & NSLEVAL & Lanczos & 3021 & {$[12]$} \\
Freund-Nachtigal & 90 & EIGLAL & Lanczos & 2375 & {$[20]$} \\
\hline
\end{tabular}

algorithms. As we have mentioned, most programs are still research codes and are subject to change. The authors of these programs reserve all rights. The reader should not judge programs by the number of Fortran lines. Some of the software are not in public release format. The line count just gives a rough feeling about how much effort was needed to write the codes.

Let us sketch the main features of each program. The first three programs in Table 2 are three different implementations based on the simultaneous iteration:

1. LOPSI by Stewart and Jennings [53] is based on bi-iteration for computing the set of eigenvalues of largest absolute magnitude of a matrix together with the corresponding left and right eigenvectors.

2. SRRIT by Bai and Stewart [6] computes a partial Schur decomposition corresponding to the eigenvalues of largest modulus instead of computing the partial eigendecomposition of LOPSI. If explicit eigenvectors are desired, they may be obtained from the Schur form, say using the LAPACK subroutine STREVC. The user guide report of SRRIT is available by anonymous ftp from thales.cs . umd. edu in the directory pub/reports. The program is available in pub/srrit.

3. EB12 by Duff and Scott [18] is the latest entry for the simultaneous iteration, which can be found in the Harwell Subroutine Library. The Chebyshev acceleration technique proposed by Saad is used in the EB12 subroutine. It also has some features such as optional computation of eigenvalues of rightmost or leftmost real part, or largest modulus.

The programs ARNLS, ARNINV and ARNUPD in Table 2 are variant implementations of the Arnoldi method. To restrict the required storage, all programs use a fixed number of steps in the Arnoldi process, and restart the process if necessary. Specifically,

1. ARNLS by Saad [42] is an implementation of the polynomial preconditioned Arnoldi method with deflation. The subroutine computes only one eigenvalue or a complex conjugate pair at a time. Deflation is then used to compute the next desired eigenpair until satisfied. For the next wanted eigenvalue a polynomial transformation is made to transform the desired eigenvalue to be very large compared to the remaining eigenvalues.

2. ARNINV by Saad [42] is an Arnoldi method with shifting-and-inverting strategy for computing the desired number of eigenvalues and associated Schur vectors closest to the shift $\sigma$ of a banded matrix $A$. The matrix $A$ is passed in banded format and then $A-\sigma I$ is factorized by LINPACK which is then used as the operator for the Arnoldi method. Although this operator may be complex, the main computations are done in real arithmetic [37]. The user can decide to use a new shift after a certain number of restarted Arnoldi when computing some eigenvalue. 
3. ARNUPD by Sorensen [49] treats the residual vector, after a run of Amoldi process, as a function of the initial vector. This initial vector is then updated through a chosen (polynomial) filter that is designed to force convergence of the residual to zero. The iterative scheme is shown to be a truncation of the standard implicitly shifted QR-iteration for the dense problem and avoids the need to explicitly restart the Arnoldi sequence.

There are two entries based on the nonsymmetric Lanczos method:

1. NSLEVAL by Cullum and Willoughby [12] is a Lanczos procedure with no reorthogonalization and no handling of possible breakdown. The procedure produces a complex symmetric tridiagonal matrix whose eigenvalue problem is solved by a modified QL algorithm.

2. EIGLAL by Freund and Nachtigal [20] is a modified Lanczos procedure with look-ahead strategy to skip over the possible breakdown. The procedure produces a Hessenberg matrix, and then its eigenproblem is solved by the QR algorithm from EISPACK.

Both implementations give rise to 'spurious' eigenvalues. In order to distinguish between 'good' and 'spurious' eigenvalues, the most common empirical way of doing this is to calculate eigenvalues of $T_{m}$ and those of $T_{m}$ with the first row and column removed. The 'good' eigenvalues repeat in these calculation, whereas the 'spurious' eigenvalues do not repeat [12].

\subsection{Simultaneous iteration versus Arnoldi versus Lanczos}

So far, we have not seen a published comprehensive comparative study of the three methods. We have seen that many factors from implementation and application problems could affect such a comparison. In theory, the fundamental advantage of the Lanczos and Arnoldi methods is that they discard no information of the generated base vectors, whereas simultaneous iteration, at each step, overwrites a set of approximation eigenvectors (or Schur vectors) with a better set. Such an advantage of the Lanczos and Arnoldi methods has been illustrated in many numerical experiments. However, a firm convergence theory has been established for simultaneous iteration [51], but there are still many questions for the Arnoldi and Lanczos algorithms [44]. In contrast with the symmetric eigenproblem, the loss of (bi)orthogonality among the computed basis (bases) of the Krylov subspaces in Arnoldi and Lanczos and its effects are not well understood. The Arnoldi method could also suffer from unbounded growth in core-storage. Although the Lanczos method only requires six or eight vectors in core-storage (for the look-ahead Lanczos scheme, it is a little high), the user must provide the vector $z=A^{T} x$, which may not be available in some applications. Without a doubt, the three methods will still continue to compete with each other in the coming years. With the growing number of applications and improvement of software the picture will be clearer.

\section{What have we learned since 1982}

Since the publication of Parlett's essay [35] (which was from a talk given at the Sparse Matrix Symposium held in Fairfield Glade, Tennessee, in 1982), we have still been seeking a better way for an old task. Nevertheless, with the contributions of many people we have made a significant advance in our expertise for the problem. 
1. BLAS: The wide popularity of the (dense or sparse) BLAS for the basic vector and matrix operations, such as the inner product, is beneficial to the software development. The programs turn out to be of simpler structure and to gain better performance with optimized BLAS.

SPARSKIT of Saad [43] is a tool to manipulate and perform simple operations with sparse matrices, such as exchange data structure, as well as basic linear algebra routines for sparse matrices. Its use in software development has been growing.

The most recent release of MATLAB includes sparse matrix storage and operation features [24].

2. The so-called algorithm independency: It means that an algorithm refers the matrix in question only through external user supplied subroutines to form the matrix-vector multiplications $A v$ and/or $A^{T} v$ (or $f(A) v$ and/or $f\left(A^{T}\right) v$, where $f(\cdot)$ is a spectral transformation function). Although this has been known for decades, today it has been further exploited and is used in terms of reverse communication [28,50]. It implies that one can develop a set of 'universal' software that is applicable to any matrix. To apply the software to a particular matrix, one simply provides a subroutine to evaluate the required matrix-vector products. For some involved applications faster algorithms can be developed for such desired matrix-vector operations.

3. Spectrum transformation (preconditioning): These techniques have been reviewed in section 5.2.

4. Acceleration techniques: The idea of acceleration techniques is to update the initial vector(s) in the restarted simultaneous iteration and Krylov subspaces methods and to force the convergence of the residual vector(s) to zero faster. These techniques have been used in simultaneous iteration and Arnoldi method [44,49].

5. Deflation technique: As a common phenomenon in all algorithms, some of the eigenvalues (and eigenvectors or Schur vectors) converge faster than the others. Therefore, deflation procedures for those convergent eigenvalues (and eigenvectors or Schur vectors) can be quite effective in terms of execution time in practice. Although the technique has been used since before 1982, for example, in [52], there is a complete study of the so-called Wielandt deflation procedure by Saad from 1985 [40]. It has become very common to include such deflation procedure in software.

6. Assessment of computed results: The nonsymmetric eigenvalue problem is harder than the symmetric counterpart, partly because of the possible extreme ill-conditioning of the problem and higher sensitivity to the roundoff error. For nonsymmetric matrices the norms of the residuals of approximate eigenpairs do not provide sufficient information to bound the error in the approximate eigenvalue. Kahan-Parlett-Jiang's theorem [30], developed in 1982, first gives a bound on the distance to the nearest matrix for which the given approximations are exact. This is the best we can expect. Such backward error analysis has served as the stopping criterion for iteration methods. The numerical practices of this bound were reported by Cullum and Willoughby in 1986 [12].

\section{Work in progress}

The nonsymmetric eigenproblem, whether small or large, dense or sparse, is one of the hardest linear algebra problems to solve effectively and accurately. For the dense eigenvalue problem, research in recent years has mostly concentrated on the parallel algorithm development. The following is an outline of some of the work in progress. 
Increasing the efficiency of the QR algorithm on the parallel computing environment is still a challenging problem. Dubrulle [17] reported the performance of the modified multishift QR algorithm on IBM 3090 machines; Henry [27] studied different strategies in the QR algorithm to improve the performance, such as the use of block methods, reducing the average stride and data movement with hybrid steps, and the use of block data structures. Van Dooren [54] proposed a prototype of block QR algorithm, but the applicability of this idea is unclear. The convergence analysis of the general $Q R$ algorithm is carried out in [55].

The great success of the divide and conquer idea for the symmetric tridiagonal eigenproblem has led several researchers to extend it to the nonsymmetric Hessenberg eigenproblem. By setting one (or more) subdiagonal element(s) of the Hessenberg matrix to zero to form independent Hessenberg eigenproblems which can be solved in parallel, and then merging the solutions of subproblems by rank-update and iterative refinement technique [16] or homotopy technique [31] to yield the solution of the original problem. Although some successful aspects have been seen, more investigation is needed to deal with the possible divergence of the iteration and identification problem, which means that we need to know, if two iterations converge to the same root, whether it is a multiple root or whether we have missed a root? Theoretical aspects of such a divide and conquer method have been discussed in recent papers by Adams and Arbenz [1], Jessup [29].

A new divide and conquer method to find the Schur decomposition by using tools such as the matrix sign function or beta function is under investigation by several groups $[32,3,5]$. There is no identification problem for the methods and is built on highly blocked matrix operations. But the use of the inverse of the original matrix and the possible significant increase in the number of the floating-point operations are the potential barrier for the idea. It will be interesting to see what happens.

The numerical analysis community is still at the stage of developing more reliable, robust and efficient sequential algorithms for the sparse eigenproblem, and of analyzing the numerical behaviors of the algorithms. Most recently, a generalization of the shift-and-invert Krylov subspace methods (Arnoldi or Lanczos) for the nonsymmetric matrix pencil eigenvalue problem has been investigated by Ruhe [39]. It uses several shifts at a time to build a rational Krylov subspace. The work is at its early stage.

A recent report by Chatelin and Godet-Thobie [10] is focused on the influence of the departure from normality of the matrix on the stability of the Arnoldi algorithm. The research being undertaken by Bai [4], and Day [14] is aiming at the error analysis of the Krylov subspaces method in the presence of the floating-point arithmetic and the effect of the loss of the (bi-)orthogonality in the practical computation.

We terminate this section with an interesting practical higher-order $\lambda$-matrix eigenvalue problems of the form

$$
\left(\lambda^{5} A_{0}+\lambda^{4} A_{1}+\lambda^{3} A_{2}+\lambda^{2} A_{3}+\lambda A_{4}+A_{5}\right) x=0
$$

The problem is from structural mechanics combined with aerodynamics [10]. Besides transforming such an eigenvalue problem to the standard eigenvalues problem, not much progress has been made concerning how to solve such $\lambda$-matrix eigenvalue problems directly and efficiently. 


\section{Conclusions}

The nonsymmetric eigenvalue problem is inherently a much harder problem than the symmetric eigenvalue problem. For small problems the QR algorithm essentially occupies the picture, although the best way to solve it on parallel computational environment is still challenging us. For large problems it is still an open research topic despite all the progress made. While numerical analysts are seeking better ways to implement existing algorithms or new algorithms, engineers will go ahead and try to solve their problems with or without help from numerical analysts. Certainly, when numerical analysts provide better tools for solving the problem, the number of applications will grow.

Finally, in honor Professors Kahan's and Parlett's 60th birthdays, we quote a conversation between Professor Kahan and a group of computer science students:

Question: Working on the problem you have been honored for (Turing award), you must have had time when you thought about throwing it all in. So, what motivated you to continue to reach your aim?

Kahan: The thought of 'throwing it all in' never occurred to me; I was motivated by curiosity, each obstacle was just another problem to analyze, understand, and then overcome.

\section{Acknowledgements}

The author thanks many colleagues and friends for providing invaluable helpful information on the nonsymmetric eigenvalue problem and sharing their programs which the author could not possibly possess. In particular, the author is grateful for the unique opportunity of visiting IMA at University of Minnesota during its 1991-1992 Applied Linear Algebra year, and being able to communicate on this subject with numerical analysis experts: Françoise Chatelin, Jane Cullum, Jim Demmel, Iain Duff, Roland Freund, Gene Golub, Anne Greenbaum, Nick Higham, Noel Nachtigal, Axel Ruhe, Youcef Saad, Danny Sorensen, Pete Stewart, Zdenek Strakos and David Watkins. The author would also like to express thanks to Nick Higham and the referees, whose comments led to improvements in the presentation, and to the editor, Jim Bunch, for improvements in the English.

\section{REFERENCES}

1. L. Adams, and P. Arbenz. Towards a divide and conquer algorithm for the real nonsymmetric eigenvalue problem, Dept. of App. Math. University of Washington, 1991.

2. E. Anderson, Z. Bai, C. Bischof, J. Demmel, J. Dongarra, J. Du Croz, A. Greenbaum, S. Hammarling, A. McKenney, S. Ostrouchov and D. Sorensen. LAPACK Users' Guide, Release 1.0, SIAM, 1992, Philadelphia, PA.

3. L. Auslander and A. Tsao. On parallelizable eigensolvers, Advances in Applied Mathematices, 13, 253-261, 1992.

4. Z. Bai. Error analysis of the Lanczos algorithm for the nonsymmetric eigenvalue problem, Math. Comp., 62, 209-226, 1994.

5. Z. Bai and J. Demmel. Design of a parallel nonsymmetric eigenroutine toolbox, Part I. In R. F. Sincovec et al., editors. Proceedings of the Sixth SIAM Conference on Parallel Processing for Scientific Computing, SIAM, Philadelphia, PA, 1993.

6. Z. Bai and G. W. Stewart. SRRIT: a Fortran Subroutine to Calculate the dominant invariant subspace of a nonsymmetric matrix, Dept. of Comp. Sci. University of Maryland, April, 1992.

7. N. E. Bixler. Stability of coating flow. Ph.D. thesis, University of Minnesota, Minneapolis, MN, 1982.

8. A. G. Boudouvis, J. L. Puchalla, L. E. Scriven and R. E. Rosensweig. Normal field instability and patterns in pools of ferrofluid. $J$. of Magnetism and Magnetic Materials, 65, 307-310, 1987. 
9. A. G. Boudouvis and L. E. Scriven. A three-dimensional pattern selection scenario, Technical Report 91/248, University of Minnesota Supercomput. Inst. 1991.

10. F. Chatelin and S. Godet-Thobie. Stability analysis and nonnormal matrices in aeronautical industries. In R. Vichnevetsky and J. Miller, editors, Proceedings of the 13th IMACS World Congress on Computation and Applied Mathematics, 1991.

11. K. L. Christodoulou and L. E. Scriven. Finding leading modes of a viscous free surface flow: an asymmetric generalized eigenproblem. J. of Scient. Comput., 3, 355-406, 1988.

12. J. Cullum and R. A. Willoughby. A practical procedure for computing eigenvalues of large sparse nonsymmetric matrices. In J. Cullum and R. A. Willoughby, editors, Large Scale Eigenvalue Problems. North-Holland, Amsterdam, 1986.

13. E. R. Davidson. Super-matrix methods. Comput. Phy. Comm., 53, 49-60, 1989.

14. D. Day. Semi-duality in the two-sided Lanczos algorithm. Ph.D. thesis, University of California at Berkeley, 1993.

15. J. Dongarra, J. Bunch, C. Moler and G. W. Stewart. LINPACK User's Guide. SIAM, Philadelphia, PA, 1979,

16. J. Dongarra and M. Sidani. A parallel algorithm for the non-symmetric eigenvalue problem. SLAM J. Sci. Comp., 14(3), 542-569, 1993.

17. A. Dubrulle. The multishift $Q R$ algorithm: is it worth the trouble? Palo Alto Scientific Center Report G320-3558x, IBM Corp., 1991.

18. I. Duff and F. A. Scott. Computing selected eigenvalues of sparse nonsymmetric matrices using subspace iteration. ACM Trans. on Math. Soft., 19, 137-159, 1993.

19. L. E. Eriksson and A. Rizzi. Computer aided analysis of the convergence to steady state of discrete approximations to the Euler equations. J. of Comput. Phys., 57, 90-128, 1985.

20. R. Freund and N. Nachtigal. An Implementation of the Look-Ahead Lanczos Algorithm for Non-Hermitian Matrices, Part II. Technical Report TR 90-46, RIACS, Nov. 1990.

21. A. Galick, T. Kerkhoven and U. Ravaioli. Iterative solution of the eigenvalue problem for a dielectric wageguide. CSRD Report 1119, University of Illinois at Urbana-Champaign, July 1991.

22. B. S. Garbow, J. M. Boyle, J. J. Dongarra and C. B. Moler. Matrix Eigensystem RoutinesEISPACK Guide Extension, Volume 51 of Lecture Notes in Computer Science. SpringerVerlag, Berlin, 1977.

23. T. J. Garratt, G. Moore and A. Spence. Two methods for the numerical detection of Hopf bifurcations. In R. Seydel et al., editors, Bifurcation and Chaos: Analysis, Algorithms, Applications. Birkhauser, Basle, 1991.

24. J. Gilbert, C. Moler and R. Schreiber. Sparse matrices in MATLAB: design and implementation. SIAM J. Mat. Anal. Appl, 13, 333-356, 1992.

25. I. Goldhirsch, S. A. Orszag and B. K. Maulik. An efficient method for computing leading eigenvalues and eigencectors of large asymmetric matrix. J. of Scient. Comput., 2, 33-58, 1987.

26. G. Golub and C. Van Loan. Matrix Computations. Johns Hopkins University Press, Baltimore, MD, second edition, 1989.

27. G. Henry. Increasing data reuse in the unsymmetric QR algorithm. Technical Report CTCTR-100. Cornell Theory Center, Cornell University, 1992.

28. N. J. Higham. Fortran codes for estimating the one-norm of a real or complex matrix, with applications to condition estimation. ACM Trans. Math. Soft., 14, 381-396. 1988.

29. E. Jessup. A case against a divide and conquer approach to the nonsymmetric eigenproblem. Technical Report ONRL/TM-11903, Oak Ridge National Laboratory, 1991.

30. W. Kahan, B. Parlett and K. Jiang. Residual bounds on approximate eigenvalues of a nonnormal matrices. SIAM J. Numer. Anal., 19, 470-484. 1982.

31. T.-Y. Li and $\mathrm{Z}$. Zeng. Homotopy-determinant algorithm for solving nonsymmetric eigenvalue problems. Math. Comp., 59(200), 483-502, 1992.

32. C-C. Lin and E. Zmijewski. A parallel algorithm for computing the eigenvalues of an unsymmetric matrix on an SIMD mesh of processors. Dept. of Comp. Sci. TRCS 91-15, University of California, Santa Barbara, July 1991.

33. A. Mahajan, E. H. Dowell and D. Bliss. Eigenvalue calculation procedure for an Euler/NavierStokes solver with applications to flows over airfoils. J. of Comput. Phys., 97, 398-413, 1991. 
34. N. Martins. Efficient eigenvalue and frequency response methods applied to power system small-signal stability studies. IEEE Trans. on Power Systems, PWRS-1, 217-226, 1986.

35. B. Parlett. The software scene in the extraction of eigenvalues from sarse matrices. SIAM J. Sci. Stat. Comput., 5, 590-604, 1984.

36. B. Parlett and $\mathrm{W}$. Heng. The method of minimal representation in 2D Ising model calculations. PAM-549, University of California, Berkeley, 1992.

37. B. Parlett and Y. Saad. Complex Shift and invert strategies for real matrices. Lin. Alg. and Appl., 88/89, 575-595, 1987.

38. A. Ruhe. Rational Krylov sequence methods for eigenvalue computations. Lin. Alg. Appl., 58, 391-405, 1984.

39. A. Ruhe, Rational Krylov algorithms for nonsymmetric Eigenvalue problems. In G. Golub, A. Greenbaum and M. Luskin, editors, Recent Advances in Iterative Methods, IMA Volumes in Mathematics and its Applications 60, pages 149-164. Springer-Verlag, New York, 1993.

40. Y. Saad. Practical use of polynomial preconditionings for the conjugate gradient method. SIAM J. Sci. Stat. Comput., 6, 865-881, 1985.

41. Y. Saad. Least squares polynomials in the complex plane and their use for solving sparse nonsymmetric linear systems. SLAM J. Num. Anal., 24, 155-169, 1987.

42. Y. Saad. Numerical solution of large nonsymmetric eigenvalue problems. Comput. Phys. Comm., 53, 71-90, 1989.

43. Y. Saad. SPARSKIT: A basic tool kit for sparse matrix computation. CSRD Report 1029, University of Illinois at Urbana-Champaign, August, 1990.

44. Y. Saad. Numerical Methods for Large Eigenvalue Problems. Halsted Press (John Wiley), New York, 1992.

45. M. Sadkane. Block-Arnoldi and Davidson methods for unsymmetric large eigenvalue problems. Numer, Math., 64, 195-211, 1993.

46. M. Sadkane. A block Arnoldi-Chebyshev method for computing the leading eigenpairs of large sparse unsymmetric matrices. Numer. Math., 64, 181-193, 1993.

47. S. Seki, T. Yamanaka and $\mathrm{K}$. Yokoyama. Two-dimensional analysis of optical waveguides with a nonuniform finite difference method. IEE Proceedings-J, 138, 123-127, 1991.

48. B. T. Smith, J. M. Boyle, J. J. Dongarra, B. S. Garbow, Y. Ikebe, V. C. Klema and C. B Moler. Matrix Eigensystem Routines-EISPACK Guide, Springer-Verlag, Berlin, 1976.

49. D. Sorensen. Implicit application of polynomial filters in a $k$-step Arnoldi method. SLAM J. Mat. Anal. Appl., 13(1), 357-385, 1992.

50. D. Sorensen and P. Vu. Private communication, 1992.

51. G. W. Stewart. Simultaneous iteration for computing invariant subspaces of non-Hermitian matrix. Numer. Math., 25, 123-126, 1976.

52. G. W. Stewart. SRRIT: a Fortran subroutine to calculate the dominant invariant subspace of a nonsymmetric matrix. Dept. of Comp. Sci. TR 154, University of Maryland, 1978.

53. W. J. Stewart and A. Jennings. Algorithm 570 LOPSI: a simultaneous iteration algorithm for real matrices. ACM Trans. on Math. Soft., 7, 230-232, 1981.

54. P. Van Dooren. Block shifts and block QR. Presentation at SIAM Conf. on Appl. Lin. Alg., Minneapolis, MN, September 1991. SIAM. Philadelphia, PA, 1991.

55. D. Watkins and L. Elsner. Convergence of algorithms of decomposition type for the eigenvalue problem. Lin. Alg. Appl., 143, 19-47, 1991. 